\title{
ANNONCES DE CONGRES ET \\ REUNIONS SCIENTIFIQUES
}

Annual meeting of the Society for the Study of Male Reproduction

DALLAS, TEXAS, USA, $1^{\text {er }}$ mai 1999

Contact : SSMR, 1111 North Plaza Drive, Suite 550, Schaumburg, Illinois 60173, USA. Tél. : 847-517-7225

$1^{\text {th }}$ World Congress on Human Reproduction

BAHIA, BRESIL, 4-8 mai 1999

Contact : Congress Secretariat, CEPARH, Rua Caetano Moura 35, Salavador 40210341, Bahia, Brésil

Tél. : 55-71-243-0244, 55-71-243-5173 - Fax : 55-71-243-9153, 55-71-339-2302

E-mail : humrepbahia@e-net.com.br

$11^{\text {th }}$ World Congress on in Vitro Fertilization and Human Reproductive Genetics

SYDNEY, AUSTRALIE, 9-14 mai 1999

Contact : Congress Secretariat, Eleanor Loveridge, Conference Action, P.O. Box 1231, North Sydney NSW, 2059, Australie

Tél. : 61-2-9956-8333 - Fax : 61-2-9956-5154

E-mail : confact@real.net.au

6èmes Journées nationales de la Société Marocaine d'Andrologie et de Sexologie (SMAS)

RABAT - HOTEL HILTON - 13-14-15 mai 1999

- Dysfonctions sexuelles

- Sperme - Fertilité et sexualité

- AMP et Vécu

- Questions d'Andrologie et de Sexologie

- Androcrinologie

- Obésité et Sexualité

- Ateliers 
Contact : Dr Aziz SMIRES

44 rue Abou Abdellah Nafii Maârif - 20100 CASABLANCA, Maroc

Tél. : (212-2) 25-95-73 / 26-59-07 - Fax : (212-2) 98-60-51

E-mail : asmires@techno.net.ma

$2^{\text {nd }}$ International Consultation on Prostate Cancer

PARIS - Palais des Congrès, Porte Maillot, 27-29 juin 1999

Web address : www.urology-publications.com

Contact : Pr Saad KHOURY

Clinique urologique (Pr Richars) Hôpital de la Pitié

83, bd de l'Hôpital 75634 PARIS CEDEX 13

Tél. : 01-42-17-71-20 - Fax : 01-42-17-71-22

E-mail : s.khoury@wanadoo.fr

$1^{\text {st }}$ International Consultation on Erectile Dysfunction

PARIS - Palais des Congrès, Porte Maillot, 1-3 juillet 1999

Web address : www.urology-publications.com

Contact : Pr Saad KHOURY (voir ci-dessus)

$1^{\text {th }}$ Annual Meeting of ESHRE

TOURS, France, 27-30 juin 1999

Contact : ESHRE Central Office, c/o Bruno van den Eede,

Van Aktenstraat 41, B-1850, Grimbergen, Belgique

Tél. : 32-2-26-09-69 - Fax : 32-2-269-56-00

E-mail : eshre@pophost.eunet.be

Second International Congress of Pan Arab Society of Andrological Sciences BEIRUT, LIBAN, 17-21 Septembre 1999

Contact : Pan Arab Society of Andrological Sciences

Fax : 202-3551742, ou 203-4833076, ou 203-4833271, ou 00961-7761942 


\section{International Workshop : Estrogens and male reproduction}

Centre des Congrès de l'Université de Calabre, RENDE, Italie, 23-24 Septembre 1999

- Expression du gène de l'aromatase dans les tissus gonadiques et extragonadiques

- Récepteurs des estrogènes dans la gonade mâle

- Les estrogènes dans le développement du testicule et la spermatogénèse

- Estrogènes et fertilité du mâle

- Aspects cliniques et thérapeutiques des estrogènes

- Anti-estrogènes et inhibiteurs de l'aromatase chez l'homme

Contact : V. PEZZI

Centro Sanitario Universita di Calabria RENDE (CS) 87036 - Italie

Tél. : (39) 984839624 ; Fax (39) 984401275

E-mail : sando@diemme.it

\section{6 ème Congrès de la Société d'Andrologie de langue française}

PARIS 9-10-11 Décembre 1999

Le programme prévoit des conférences, des tables rondes, des communications orales et affichées sur panneaux.

Principales sessions

-Gamètes et molécules de signalisation

-Tumeurs testiculaires

-Cryoconservation des gamètes et du tissu germinal

-Vieillissement génito-sexuel masculin

-Andrologie et handicap

Contacts : Dr F.GIULIANO, Urologie

CHU de Bicètre, 78, rue du Général Leclerc 94275 Le Kremlin Bicètre

Tél. : 01.45.21.36.98; Fax : 01.45.21..36.76

Pr. S.HAMAMAH, Biologie de la Reproduction

Hôpital A.Béclère, 157, rue de la Porte de Trivaux 92141 Clamart

Tél : 01.45.37.49.79; Fax : 01.45.37.42.92 
- The Second World Congress on the Aging Male

GENEVE, SUISSE, 9-13 février 2000

Contact : Sally Preece, ISSAM Secrétariat

Tél. : 44-15242-72084

Web : http:/www.kenes.co.il

$1^{\text {st }}$ European Congress of Andrology

L'AQUILA, Italie, 26-28 mars 2000

Contact : Prof. G. Forti, Andrology, Universita di Firenze,

Viale Morgagni 85, I-50134, Firenza, Italie

Tél. : 39-5543-77854 Fax : 39-5543-77220

E-mail : g.forti@dfc.uni.it 